\title{
Metabolic Consequences Of Obesity On The Hypercoagulable State Of Polycystic Ovary Syndrome: A Risk For Severe SARS- Cov-2 Infection?
}

\author{
Abu Saleh Md Moin \\ Qatar Biomedical Research Institute \\ Thozhukat Sathyapalan \\ Hull York Medical School \\ Ilhame Diboun \\ Hamad Bin Khalifa University \\ Mohamed Elrayess \\ Qatar University \\ Alexandra E Butler ( $\square$ aeb91011@gmail.com ) \\ Qatar Biomedical Research Institute \\ Stephen L Atkin \\ Royal College of Surgeons in Ireland, Bahrain
}

\section{Research Article}

Keywords: Polycystic ovary syndrome, D-dimer, Fibrinogen, Fibrin degeneration products

Posted Date: September 16th, 2020

DOI: https://doi.org/10.21203/rs.3.rs-76784/v1

License: (c) (i) This work is licensed under a Creative Commons Attribution 4.0 International License. Read Full License 


\section{Abstract}

Introduction: Polycystic ovary syndrome (PCOS) women have a hypercoagulable state and are also at high risk for severe COVID-19 leading to thromboembolic complications and increased mortality; however, whether this is intrinsically due to PCOS or, alternatively, a consequence of its metabolic complications is unclear.

Methods: We determined plasma coagulation pathway protein levels in PCOS $(n=146)$ and control $(n=97)$ women recruited to a PCOS biobank. Circulating levels of a panel of 18 clotting pathway proteins were determined by Slow Off-rate Modified Aptamer (SOMA)-scan plasma protein measurement.

Results: Cohorts were age matched, though PCOS had elevated body mass index (BMI) $(\mathrm{p}<0.001)$, insulin $(\mathrm{p}<0.001)$ and $\mathrm{C}$-reactive protein (CRP) $(p<0.0001)$. Eight pro-coagulation proteins were elevated in PCOS: plasminogen activator inhibitor-1 (PAl-1) $(\mathrm{p}<0.0001)$, fibrinogen ( $<<0.01)$, fibrinogen gamma chain $(p<0.0001)$, fibronectin $(p<0.01)$, von Willebrand factor $(p<0.05), D-d i m e r(p<0.0001), P$ selectin $(p<0.05)$, and plasma kallikrein $(p<0.001)$. However, two anticoagulant proteins, vitamin K-dependent protein-S $(p<0.0001)$ and heparin cofactor-II $(p<0.001)$ were elevated and prothrombin was decreased $(p<0.05)$. CRP, as a marker of inflammation, and insulin resistance (HOMA-IR) correlated with 11 and 6 of the clotting proteins, respectively $(p<0.05)$. When matched for BMl<25 (16 PCOS, 53 controls) HOMA-IR remained elevated $(p<0.05)$ and heparin cofactor-II was increased $(p<0.05)$. In a multivariate analysis accounting for inflammation, insulin resistance and BMI, there was no correlation of PCOS with any of the coagulation proteins.

Conclusion: The hypercoagulable State in PCOS can be fully accounted for by BMI, inflammation and insulin resistance suggesting that only obese PCOS women would be predisposed to an enhanced risk for severe COVID-19-related disease.

\section{Introduction}

PCOS has been recognized as a reproductive-metabolic disorder given the excess prevalence of type 2 diabetes, hypertension, and cardiovascular diseases in this population at a later stage in life (1). Polycystic ovary syndrome (PCOS) patients have increased platelet aggregation and decreased plasma fibrinolytic activity, resulting in a prothrombotic propensity $(2,3)$. Elevated coagulation markers have been reported in PCOS in comparison to controls (4) and the coagulation parameters including prothrombin time, thrombin time and fibrin degradation products may be predictive of PCOS (5). It has been reported that coagulation proteins such as thrombin-activatable fibrinolysis inhibitor, PAI-1, D-dimer, Antithrombin III and thrombomodulin are significantly increased in women with PCOS compared with age- and BMI-matched controls (4). This suggests that PCOS, independent of its metabolic features, may be a risk factor for a hypercoagulable state, and thus PCOS women may be at high risk for severe COVID-19 infection $(6,7)$.

COVID-19 patients have a hypercoagulable state resulting in thromboembolic complications and increased mortality. Whilst the pathogenesis is not fully understood, evidence suggests a combination of endothelial injury, blood stasis and changes in circulating prothrombotic factors such as elevated D-dimer, factor VIII, fibrinogen and fibrin degeneration products and reduced antithrombin levels (8).

This study was undertaken to determine the parameters contributing to the hypercoagulable state reported for PCOS and the potential risk of developing severe COVID19 disease.

\section{Materials And Methods}

We determined plasma coagulation pathway protein levels in PCOS $(n=146)$ and control $(n=97)$ women recruited to a PCOS biobank (ISRCTN70196169). The diagnosis of PCOS was based on at least two out of three of the diagnostic criteria of the Rotterdam consensus as detailed previously (9); namely clinical and biochemical evidence of hyperandrogenism (Ferriman-Gallwey score $>8$; free androgen index $>4$, total testosterone $>1.5 \mathrm{nmol} / \mathrm{L}$ ), oligomenorrhea or amenorrhoea and polycystic ovaries on transvaginal ultrasound. Nonclassical 21-hydroxylase deficiency, hyperprolactinemia, Cushing's disease and androgen secreting tumors were excluded by appropriate tests. The baseline study measurements have been described in detail previously (10) and the demographic data for the PCOS and control women is shown in Table 1. All the control women had regular periods, no clinical or biochemical hyperandrogenism, no polycystic ovaries on ultrasound, no significant background medical history and none of them were on any medications including oral contraceptive pills or over the counter medications. 
Circulating levels of clotting pathway proteins were determined by Slow Off-rate Modified Aptamer (SOMA)-scan plasma protein measurement, the details of which have been previously reported (11). Normalization of raw intensities, hybridization, median signal and calibration signal were performed based on the standard samples included on each plate, as previously described (12).

We used version 3.1 of the SomaScan Assay, specifically targeting those proteins involved in the coagulation pathways in the SomaScan panel of 18 proteins: antithrombin III, heparin cofactor 2, fibrinogen gamma chain, D-Dimer, P-selectin, fibronectin, fibronectin fragment 3 , fibronectin fragment 4, vitamin $\mathrm{K}$ dependent protein $\mathrm{S}$, alpha 2 antiplasmin, fibrinogen, von Willebrand factor, plasma kallikrein, prothrombin, coagulation factor Xa, tissue factor, coagulation factor XI and angiostatin (Table 2).

\section{Statistics}

Measured protein data were log transformed to ascertain normality. Proteins were regressed on the continuous variables CRP, HOMA-IR and BMI in separate models to assess the extent of association with each trait. A multivariate linear model incorporating all three traits and PCOS status was performed to evaluate the relationship between the measured proteins and PCOS whilst correcting for the traits. All analyses were performed using R version 4. P values were corrected for multiple testing using the false discovery rate (FDR).

\section{Results}

Cohorts were age matched, thoughPCOS had elevated BMI $(p<0.001)$, fasting glucose $(p<0.05)$, insulin $(p<0.001)$, C-reactive protein $(p<0.0001)$ and platelet number $(p<0.01)$.

Pro-coagulation proteins elevated in PCOS are shown in Figure 2 and include plasminogen activator inhibitor-1 (PAI-1) (2259 \pm 137 vs $1457 \pm 107$ RFU, PCOS vs control, $p<0.0001$ ), fibrinogen ( $177423 \pm 2108$ vs $169230 \pm 2425$ RFU, PCOS vs control, $p<0.01$ ), fibrinogen gamma chain ( $63118 \pm 946$ vs $57328 \pm 830 \mathrm{RFU}, \mathrm{p}<0.0001)$, fibronectin $(24594 \pm 2627$ vs $16041 \pm 698 \mathrm{RFU}, \mathrm{p}<0.01)$, von Willebrand factor (19849 \pm 3038 vs $13159 \pm 595$ RFU, $p<0.05)$, D-dimer (13860 \pm 185 vs $12708 \pm 172$ RFU, $p<0.0001)$, P-selectin (13843 \pm 317 vs $12660 \pm 412 \mathrm{RFU}, p<0.05)$, and plasma kallikrein ( $24868 \pm 376$ vs $22981 \pm 447 \mathrm{RFU}, p<0.001)$. Prothrombin levels were decreased in PCOS $(161458 \pm 1275$ vs $165233 \pm 1958$ RFU, $p<0.05)$ and the anticoagulant vitamin K-dependent protein $S(4403 \pm 69$ vs $3989 \pm 59$ RFU, $p<0.0001)$ and heparin cofactor II (HCII) $(4156 \pm 64$ vs $3821 \pm 63$ RFU, $p<0.001)$ were increased (Figure 1). Significant correlations of coagulation proteins with body mass index are shown in Table 2 and include antithrombin III $(p<0.0001)$, fibrinogen gamma chain $(p<0.0001)$, vitamin $K$ dependent protein $S(p<0.001)$, D-dimer $(p<0.001)$, fibronectin $(p<0.001)$, prothrombin $(p<0.01)$, heparin cofactor $2(p<0.01)$, fibrinogen $(p<0.05)$, angiostatin $(p<0.05)$, fibrinogen fragment $3(p<0.05)$ and P-selectin $(p<0.05)$. Significant correlations of coagulation proteins with CRP, as a marker of inflammation, are shown in Table 2, that included antithrombin III $(p<0.0001)$, heparin cofactor $2(p<0.0001)$, fibrinogen gamma chain $(p<0.0001)$, D-dimer $(p<0.0001)$, P-selectin $(p<0.001)$, fibronectin $(p<0.001)$, and its fragments 3 and $4(p<0.01$, respectively), vitamin $K$ dependent protein $S$, alpha 2 antiplasmin and fibrinogen $(p<0.05$, respectively). Significant correlations of coagulation proteins with insulin resistance, as determined by HOMA-IR, were also seen for antithrombin III ( $p<0.0001)$, heparin cofactor $2(p<0.001)$, P-selectin $(p<0.0001)$, fibronectin $(p<0.01)$, vitamin $K$ dependent protein $\mathrm{S}$ and alpha 2 antiplasmin ( $<0.05$, respectively) (Table 2$)$. However, in a multivariate analysis accounting for BMI, inflammation (CRP) and insulin resistance (HOMA-IR), there was no correlation with the coagulation proteins (Table 2).

To eliminate the confounding effect of obesity, a subset of women with BMI $\leq 25 \mathrm{~kg} / \mathrm{m}^{2}$ (16 PCOS and 53 controls) were compared. Here, HOMA-IR remained elevated in PCOS (1.6 \pm 1.2 vs $1.1 \pm 0.5, p<0.05)$, CRP did not differ, whilst heparin cofactor 2 (3979 \pm 649 vs $3613 \pm 585 \mathrm{RFU}, \mathrm{p}<0.05)$ was elevated in the normal weight PCOS group.

\section{Discussion}

These data show that the hypercoagulable state in PCOS can be completely accounted for by BMI and its associated inflammation, and enhanced insulin resistance. In comparison to the normal controls, overall 10 pro-coagulation proteins were elevated in PCOS; plasminogen activator inhibitor-1 (PAl-1), fibrinogen, fibrinogen gamma chain, fibronectin, von Willebrand factor, D-dimer, Pselectin, plasma kallikrein. anticoagulant vitamin K-dependent protein S and heparin cofactor II, whilst prothrombin was decreased. These results are in accord with others who have reported changes in coagulation proteins in PCOS $(4,13)$, but underlying 
pathophysiology has not been previously described and shows that in PCOS alterations in the coagulation factors appears complex and multifactorial. When normal weight (BMI 25$)$ PCOS patients were compared with normal weight control subjects, insulin resistance remained elevated and heparin cofactor 2 , that is protective and inactivates thrombin in tissues, differed. These data are in accord with the association of heparin cofactor 2 with insulin resistance (14), indicating that normal weight PCOS subjects likely have no additional risk associated with a hypercoagulable state; however, obesity with associated inflammation markedly exaggerates the hypercoagulable state with an increased number of clotting parameters altered. The multivariate analysis showed that all of the changes in the coagulation proteins could be accounted for by BMI, inflammation and insulin resistance.

It is well recognized that obesity causes inflammation and increased insulin resistance $(15,16)$ and is associated with changes in coagulation parameters. For example, fibronectin is correlated to BMI (17) and obesity is associated with increased PAI-1 in PCOS (18). Others have reported, using a repeated fibrin formation and degradation functional assay, that "overall hemostatic potential" was BMI-dependent and not associated with PCOS (19). Central fat mass has been associated with fibrinogen, CRP, coagulation factor XIII, waist-to-hip ratio, plasminogen, PAI-1, plasmin inhibitor, and thrombin activatable fibrinolysis inhibitor (20)

Conversely, thrombin-activatable fibrinolysis inhibitor, PAI-1, D-dimer, Antithrombin III and thrombomodulin were reported to be significantly increased in women with PCOS compared with age- and BMI-matched controls, suggesting that alterations in these proteins are BMI-independent and due to other factors such as inflammation and insulin resistance, as reported here (4).

Inflammation (CRP) correlated significantly with antithrombin III, heparin cofactor 2, fibrinogen gamma chain, D-dimer, P-selectin, fibronectin, and its fragments 3 and 4, vitamin $\mathrm{K}$ dependent protein S, alpha 2 antiplasmin and fibrinogen. Inflammation crosstalk with coagulation leading to increased coagulopathy is well recognized; however, with the initiation of coagulation, the coagulation proteases may then modulate the inflammatory response $(21,22)$. In PCOS, both CRP and fibrinogen are predicted by BMI in accord with obesity initiating the increased inflammation (23) and particularly CRP, PAI-1, D-dimer, Antithrombin III with central fat mass as noted above (20).

In this study, insulin resistance (HOMA-IR) correlated with Antithrombin III, heparin cofactor 2, P-selectin, fibronectin, vitamin K dependent protein $\mathrm{S}$ and alpha 2 antiplasmin. It is recognized that insulin resistance is associated with enhanced thrombogenesis (24); however, it is difficult to determine the contribution of insulin resistance alone to its association with obesity and inflammation metabolic syndrome lipid parameters (25-27).

As noted above, there are reports of changes in coagulation proteins in PCOS $(4,13)$ and changes in functional assays $(2,3)$; however, conversely others have not found changes in the coagulation proteins between PCOS and controls (28). It can be seen from the data presented here that the likely reason for these discrepancies are due to the patient population being studied with the results dependent on the degree of obesity, inflammation and insulin resistance present. In addition, the PCOS phenotype may have an important role, with those having all three of the diagnostic criteria exhibiting the metabolic phenotype with increased insulin resistance in comparison to those with only two of the three diagnostic criteria (29)

The hypercoagulation state is in homeostasis with the pro-coagulation protein changes seen here in PCOS being balanced by the reduction in prothrombin and increased vitamin K-dependent protein S and heparin cofactor Il that we also report; however, COVID19 disease may shift this balance towards a hyper-procoagulant state. Patients infected with COVID-19 with acute respiratory distress syndrome (ARDS) show a procoagulant pattern of coagulation markers and elevation of fibrinogen and fibrin degradation products (D-dimers) which could drive organ failure and death (8).

Limitations of this study include that it was a cross sectional study but this was mitigated by the large number of subjects. In addition, only the proteins involved in the coagulation pathways were measured and no functional assays were undertaken in this study.

In conclusion, thehypercoagulable state in PCOS can be fully accounted for by BMI, inflammation and insulin resistance, suggesting that only obese PCOS women would be predisposed to an enhanced risk for severe COVID-19-related disease.

\section{Declarations}


Ethics approval and consent to participate: The Newcastle \& North Tyneside Ethics committee approved this study. All patients gave written informed consent.

Consent for publication: All authors gave their consent for publication.

Availability of data and materials: All the data for this study will be made available upon reasonable request to the corresponding author.

Conflict of interests: No authors have any conflict of interest or competing interests to declare.

Funding: No funding was received to perform this study.

Author contribution statement: ASMM and AEB analyzed the data and wrote the manuscript. TS supervised clinical studies and edited the manuscript. ID performed the statistical analysis. SLA and MAE contributed to study design, data interpretation and the writing of the manuscript. All authors reviewed and approved the final version of the manuscript. Alexandra E Butler is the guarantor of this work.

\section{References}

1. Salley KE, Wickham EP, Cheang KI, Essah PA, Karjane NW, Nestler JE. Glucose intolerance in polycystic ovary syndrome--a position statement of the Androgen Excess Society. J Clin Endocrinol Metab. 2007;92(12):4546-56.

2. Mak W, Dokras A. Polycystic ovarian syndrome and the risk of cardiovascular disease and thrombosis. Semin Thromb Hemost. 2009;35(7):613-20.

3. Yildiz BO, Haznedaroğlu IC, Kirazli S, Bayraktar M. Global fibrinolytic capacity is decreased in polycystic ovary syndrome, suggesting a prothrombotic state. The Journal of clinical endocrinology and metabolism. 2002;87(8):3871-5.

4. Oral B, Mermi B, Dilek M, Alanoğlu G, Sütçü R. Thrombin activatable fibrinolysis inhibitor and other hemostatic parameters in patients with polycystic ovary syndrome. Gynecol Endocrinol. 2009;25(2):110-6.

5. Sun $Q$, Yang $Y$, Peng $X$, Zhang $Y$, Gao $Y$, Wang F, et al. Coagulation parameters predictive of polycystic ovary syndrome. European journal of obstetrics, gynecology, and reproductive biology. 2019;240:36-40.

6. Moin ASM, Sathyapalan T, Atkin SL, Butler AE. Renin-Angiotensin System Overactivation in Polycystic Ovary Syndrome, a Risk for SARS-CoV-2 Infection? Metabolism open. 2020:100052.

7. Kyrou I, Karteris E, Robbins T, Chatha K, Drenos F, Randeva HS. Polycystic ovary syndrome (PCOS) and COVID-19: an overlooked female patient population at potentially higher risk during the COVID-19 pandemic. BMC medicine. 2020;18(1):220.

8. Ranucci M, Ballotta A, Di Dedda U, Bayshnikova E, Dei Poli M, Resta M, et al. The procoagulant pattern of patients with COVID19 acute respiratory distress syndrome. Journal of Thrombosis and Haemostasis. 2020.

9. Sathyapalan T, Al-Qaissi A, Kilpatrick ES, Dargham SR, Atkin SL. Anti-Mullerian hormone measurement for the diagnosis of polycystic ovary syndrome. Clinical endocrinology. 2017.

10. Sathyapalan T, Al-Qaissi A, Kilpatrick ES, Dargham SR, Adaway J, Keevil B, et al. Salivary testosterone measurement in women with and without polycystic ovary syndrome. Sci Rep. 2017;7(1):3589.

11. Kahal H, Halama A, Aburima A, Bhagwat AM, Butler AE, Grauman J, et al. Effect of induced hypoglycemia on inflammation and oxidative stress in type 2 diabetes and control subjects. Sci Rep. 2020;10(1):4750.

12. Kraemer S, Vaught JD, Bock C, Gold L, Katilius E, Keeney TR, et al. From SOMAmer-based biomarker discovery to diagnostic and clinical applications: a SOMAmer-based, streamlined multiplex proteomic assay. PLoS One. 2011;6(10):e26332.

13. Targher G, Zoppini G, Bonora E, Moghetti P. Hemostatic and fibrinolytic abnormalities in polycystic ovary syndrome. Semin Thromb Hemost. 2014;40(5):600-18.

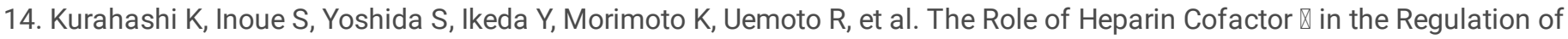
Insulin Sensitivity and Maintenance of Glucose Homeostasis in Humans and Mice. Journal of atherosclerosis and thrombosis. 2017;24(12):1215-30.

15. Saltiel AR, Olefsky JM. Inflammatory mechanisms linking obesity and metabolic disease. The Journal of clinical investigation. 2017;127(1):1-4. 
16. Shoelson SE, Herrero L, Naaz A. Obesity, inflammation, and insulin resistance. Gastroenterology. 2007;132(6):2169-80.

17. Nácul AP, Andrade CD, Schwarz P, de Bittencourt PI, Jr., Spritzer PM. Nitric oxide and fibrinogen in polycystic ovary syndrome: associations with insulin resistance and obesity. European journal of obstetrics, gynecology, and reproductive biology. 2007;133(2):191-6.

18. Atiomo WU, Fox R, Condon JE, Shaw S, Friend J, Prentice AG, et al. Raised plasminogen activator inhibitor-1 (PAl-1) is not an independent risk factor in the polycystic ovary syndrome (PCOS). Clinical endocrinology. 2000;52(4):487-92.

19. Rakusa M, Jensterle M, Božič-Mijovski M, Janez A. Increased Coagulation and Decreased Fibrinolysis as Measured with Overall Hemostatic Potential Are Dependent on BMI and Not Associated with PCOS. Metabolic syndrome and related disorders. 2017;15(4):194-8.

20. Godtfredsen ACM, Sidelmann JJ, Gram JB, Andersen M, Glintborg D. Fibrin lysability is associated with central obesity and inflammation in women with polycystic ovary syndrome. Acta Obstet Gynecol Scand. 2020;99(8):1078-84.

21. Levi M, van der Poll T. Inflammation and coagulation. Crit Care Med. 2010;38(2 Suppl):S26-34.

22. Esmon CT. The interactions between inflammation and coagulation. Br J Haematol. 2005;131(4):417-30.

23. Mažibrada I, Djukić T, Perović S, Plješa-Ercegovac M, Plavšić L, Bojanin D, et al. The association of hs-CRP and fibrinogen with anthropometric and lipid parameters in non-obese adolescent girls with polycystic ovary syndrome. J Pediatr Endocrinol Metab. 2018;31(11):1213-20.

24. Baalbaki HA, Bell DS. Insulin resistance and thrombogenesis: recent insights and therapeutic implications. Endocr Pract. 2007;13(6):679-86.

25. Lallukka S, Luukkonen PK, Zhou Y, Isokuortti E, Leivonen M, Juuti A, et al. Obesity/insulin resistance rather than liver fat increases coagulation factor activities and expression in humans. Thromb Haemost. 2017;117(2):286-94.

26. Samad F, Ruf W. Inflammation, obesity, and thrombosis. Blood. 2013;122(20):3415-22.

27. Ragab A, Abousamra NK, Higazy A, Saleh O. Relationship between insulin resistance and some coagulation and fibrinolytic parameters in patients with metabolic syndrome. Lab Hematol. 2008;14(1):1-6.

28. Kebapcilar L, Taner CE, Kebapcilar AG, Sari I. High mean platelet volume, low-grade systemic coagulation and fibrinolytic activation are associated with androgen and insulin levels in polycystic ovary syndrome. Arch Gynecol Obstet. 2009;280(2):18793.

29. Neven ACH, Laven J, Teede HJ, Boyle JA. A Summary on Polycystic Ovary Syndrome: Diagnostic Criteria, Prevalence, Clinical Manifestations, and Management According to the Latest International Guidelines. Semin Reprod Med. 2018;36(1):5-12.

\section{Tables}

Table 1: Demographics, baseline, hormonal and metabolic parameters of the PCOS subjects and controls

\begin{tabular}{|l|c|c|c|}
\hline & PCOS (n=146) & Controls (n=97) & P-value \\
\cline { 2 - 4 } Baseline demographics & Mean (SD) & Mean (SD) & \\
\hline Age (years) & $29.1(6.1)$ & $29.6(6.5)$ & 0.09 \\
\hline BMI (Kg/m) & $34.1(7.5)$ & $26.7(6.6)$ & $<0.0001$ \\
\hline Weight $(\mathrm{Kg})$ & $96.5(23.7)$ & $74.4(18.4)$ & $<0.0001$ \\
\hline Insulin (IU/ml) & $10.2(6.1)$ & $6.2(3.2)$ & 0.001 \\
\hline HOMA-IR & $3.8(0.6)$ & $1.6(0.2)$ & $<0.005$ \\
\hline CRP (mg/L) & $4.4(4.2)$ & $2.4(3.9)$ & 0.0008 \\
\hline SHBG (nmol/L) & $42.5(39.6)$ & $77.5(78.4)$ & 0.0003 \\
\hline Testosterone (nmol/l) & $1.6(1.0)$ & $1.05(0.48)$ & $<0.0001$ \\
\hline
\end{tabular}

BMI - Body Mass Index; HOMA-IR - Homeostasis model of assessment - insulin resistance; CRP- C reactive protein; $S H B G$ - sex hormone binding globulin 
Table 2. Correlation of coagulation proteins with (A) Body mass index (BMI). (B) inflammation (C reactive protein; $\mathrm{CRP}$ ) and (C) insulin resistance (HOMA-IR). (D) shows the results of the multivariate analysis taking into account BMI, CRP and HOMA-IR.

A. BMI

Antithrombin.III
Fibrinogen gamma
chain
Vitamin.K
dependent protein
S
D.dimer

Fibronectin

Prothrombin

Heparin.cofactor.2

Fibrinogen

Angiostatin

Fibronectin

Fragment.3

P.selectin

Alpha.2

antiplasmin

Fibronectin

Fragment.4

Tissue.Factor

von.Willebrand

factor

Coagulation factor

$\mathrm{Xa}$

Coagulation

Factor.XI

Plasma kallikrein
B. CRP

$\mathrm{p}$ value

$<0.00001$

0.00004

III

Antithrombin

Heparin

cofactor.2

Fibrinogen

0.00066

0.00077

gamma chain

D.dimer

0.00077

P.selectin

0.00123

P.selectin

0.00732

Fibronectin

Fibronectin

0.00864

Fragment.3

Fibronectin

0.01267

Fragment.4

Vitamin K

dependent

0.01444

protein.S

Alpha.2

0.02490

antiplasmin

0.01838

0.00144

0.00573

$\mathbf{0 . 0 0 7 1 5}$ Fragment.3

Plasma

kallikrein

0.18003

Tissue

Factor

0.19878

0.02004

0.03856

Fibrinogen

0.02176

von

Willebrand

0.15769

factor

Plasma

0.18185

0.19238

kallikrein

Prothrombin

Coagulation

0.26450

factor.Xa

Fibrinogen

gamma

chain

0.49408

Tissue.Factor

Coagulation

0.65951

Factor.XI

0.96869

Angiostatin
0.12158

0.18463

0.19448

0.22468

0.71352

0.73179

Fibronectin

Fragment.4

D.dimer

Angiostatin

Coagulation

Factor.XI

Coagulation

factor.Xa

0.80849

von

Willebrand

factor

0.73591

Fibrinogen

0.31789
0.23928

Fibronectin

Fragment.3

0.07030

Plasma

kallikrein

0.08567

Fibronectin

Fragment.4

0.12159

Vitamin.K

dependent

protein.S

0.13821

von Willebrand

factor

0.13962

Coagulation

factor.Xa

0.18692

Antithrombin.III 0.18920

Fibronectin

0.24267

Tissue.Factor

0.39938

Angiostatin

0.41172

Prothrombin $\quad 0.41800$

Alpha. 2

antiplasmin

0.41905

Heparin

cofactor 2

0.58118

Coagulation

Factor.XI

0.70381

P.selectin

0.70626

\section{Figures}


A

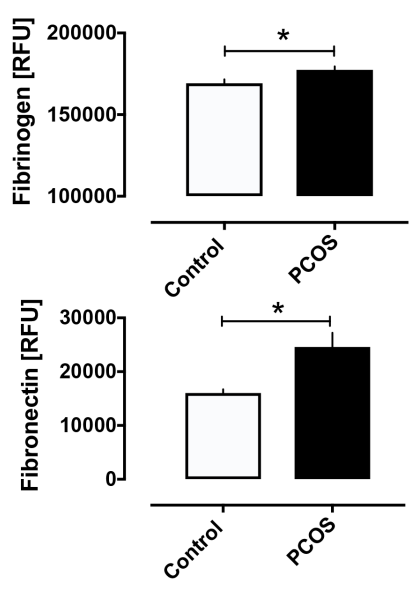

E

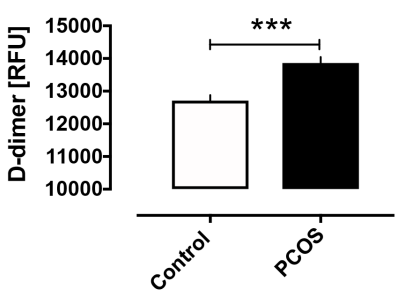

G

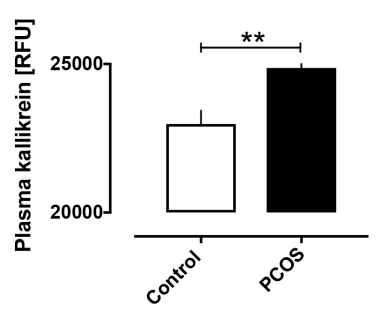

I

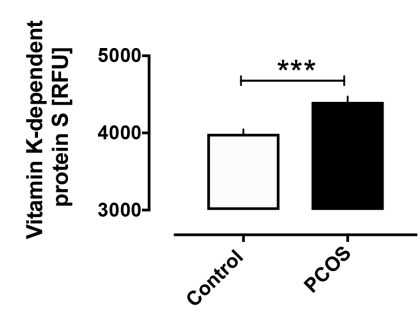

$\mathrm{B}$

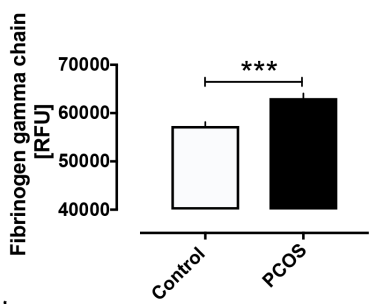

D

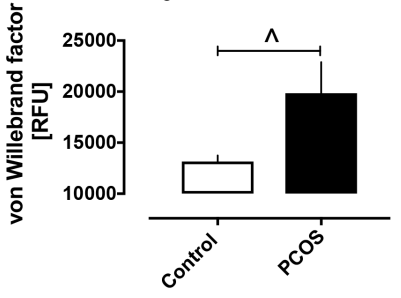

$\mathrm{F}$

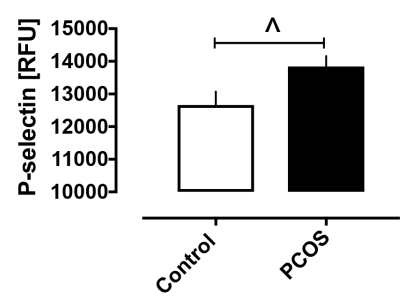

$\mathrm{H}$
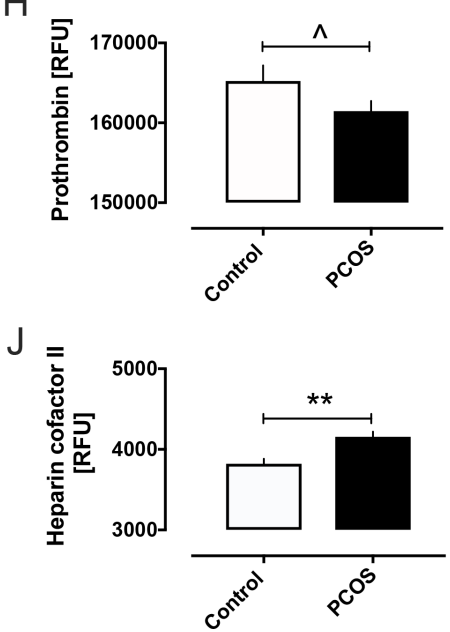

Figure 1

Figure 1. Clotting pathway proteins in women with and without polycystic ovary syndrome (PCOS). Levels of plasma fibrinogen (A), fibrinogen gamma chain (B), fibronectin (C), von Willebrand factor (D), D-dimer (E), P-selectin (F), plasma kallikrein (G) and prothrombin $(H)$ in women with and without polycystic ovary syndrome (PCOS). RFU, relative fluorescent units. ${ }^{\wedge} p<0.05,{ }^{\star} p<0.01$, ${ }^{* *} p<0.001, * * * p<0.0001$. 